La literatura oral: una apología del concepto. Parte I: La falsa analogía

\title{
LA LITERATURA ORAL: UNA APOLOGÍA DEL CONCEPTO. PARTE I: LA FALSA ANALOGÍA ${ }^{1}$
}

\author{
Vanessa Franco Ramírez \\ Universidad CES / Universidad Católica de Oriente (Colombia) \\ vanessa.franco.ramirez91@gmail.com
}

Recibido: 12/08/2020 - Aprobado: 28/09/2020 - Publicado: 15/04/2021

DOI: doi.org/10.17533/udea.lyl.n79a25

Resumen: El presente trabajo busca evidenciar la hipertrofia conceptual de la que sufre la literatura oral. Esto con la intención de revisar y fortalecer algunas de las ideas base que han venido fundamentando muchas de las investigaciones frente a este fenómeno. A través de la metodología hermenéutica, se presenta una revisión analítico-argumentativa de la premisa de la que se parte para no aceptar el concepto de literatura oral: la analogía de Walter Ong. Después, se explica la falacia por falsa analogía que constituye esta idea y, finalmente, se presenta la discusión acerca de la posible pertinencia de la nominalización literatura oral.

Palabras clave: literatura oral; hipertrofia conceptual; oralidad; escritura; estudios literarios.

\section{ORAL LITERATURE: AN APOLOGY OF THE CONCEPT. PART I: THE FALSE ANALOGY}

\begin{abstract}
This article intends to unveil the conceptual hypertrophy that is suffered by oral literature with the aim of analyze and strengthen the basic ideas that have been the foundation of many of the researches around the phenomenon. Through the hermeneutical methodology, an argumentative analysis of the premise is presented, which constitutes the starting point to reject the idea of oral literature: Walter Ong's analogy. Afterwards, the false analogy that comes along Ong's proposal and the discussion about the possible pertinence of the nominalization oral literature is explained.
\end{abstract}

Key words: oral literature; conceptual hypertrophy; orality; literacy; literary studies.


esta investigación surgieron tres productos. En el primero, se explicó y se evidenció la hipertrofia conceptual que vive la literatura oral; en el segundo, se analizó la pertinencia de las formas que se han defendido para reconceptualizar este fenómeno; y en el tercero, se aclaró por qué el concepto de literatura oral es pertinente y por qué esta debería ser estudiada desde la perspectiva de los estudios literarios. El presente artículo corresponde al primero de estos trabajos, que se constituye como el comienzo del camino para redefinir el concepto y fortalecer las ideas que fundamentan el estudio de la literatura oral.

Editores 
La literatura oral: una apología del concepto. Parte I: La falsa analogía

«Folklore is the womb of literature; literature is born of folklore»

(Propp, 1984. p. 14).

\section{Introducción: la hipertrofia conceptual}

$\mathrm{E}$

n la actualidad, el mundo académico está experimentando una problemática diametralmente diferente a la que experimentaron, por ejemplo, los primeros filósofos griegos. Hace algunos milenios, lo que se vivía al tratar de pensar por medio de la lengua era una sensación de cojera²: faltaban conceptos y sobraban objetos de estudio; por el contrario, lo que hoy se experimenta es algo que se podría denominar hipertrofia conceptual: los objetos de estudio están siendo sobre-conceptualizados.

Esto se genera, la mayoría de las veces, porque los investigadores que se dedican a las ciencias humanas - a diferencia de aquellos que estudian las ciencias naturales - suelen realizar sus observaciones desde una complejidad analítica que tiene relación con el hecho de que nuestros objetos de estudio están más allá de la percepción, son esencialmente psíquicos, y para ellos es un poco más complejo construir instrumentos y definir perspectivas de análisis. Esta dificultad surge de la condición de no poder percibir el objeto de estudio en tiempo real o a través de alguno de los sentidos; tiene que hacerse desde la propia configuración del pensamiento, que trata de analizar fenómenos que allí mismo se producen y que, además, ocupan espacios temporales muy amplios o difíciles de delimitar.

La consecuencia de esto es que los investigadores terminan sobrenominalizando, debido a que se tienden a confundir los estados o características del objeto de estudio con objetos diferentes, o debido a que se enfocan en diferenciar tajantemente dos conceptos fundamentándose en argumentos formalistas antes que esencialistas. Un caso muy claro de este fenómeno puede ser encontrado en el campo de la teoría del lenguaje, en el que diversos investigadores suelen ocuparse de los mismos objetos cayendo en la sobrenominalización o hipertrofia conceptual.

Por ejemplo, al tratar de adentrarse en la disciplina que estudia el comportamiento de los signos, uno se puede encontrar con explicaciones como esta:

El término semiología concurre con el de semiótica para designar la teoría del lenguaje [...] [So]lo hacia 1970, el contenido metodológico de la semiología y de la semiótica se diferenció progresivamente [...] [;] Hjelmslev [...] entiende por semiología la metasemiótica [...] [.] Se ahonda así la fosa entre la semiología - que considera que las lenguas naturales sirven como instrumentos de paráfrasis para describir objetos semióticos- y la semiótica - cuya primera tarea es construir un metalenguaje apropiado- (Greimas \& Courtés, 2006, pp. 361-364).

\footnotetext{
2. Evidenciada en las producciones filosóficas de los primeros pensadores, quienes debían preocuparse — antes que nada_ por nombrar los fenómenos y las características que iban a analizar en sus investigaciones. Esta situación es ilustrada claramente por Miguel Candel Sanmartín en su introducción al primer tratado de la lógica aristotélica: «[s]us limitaciones [las de Aristóteles], obvias para cualquier lógico actual, derivan fundamentalmente de que el grado de reflexión posible en su época sobre el lenguaje y el pensamiento (los dos polos de toda lógica) no podía ir más allá del marco impuesto por el lenguaje natural» (Aristóteles, 1982, p. 9).
} 
La literatura oral: una apología del concepto. Parte I: La falsa analogía

Evidentemente, la teoría de los signos está sobre-nominalizada y esto se deriva de la insistencia diferenciadora entre dos términos que están buscando dar a entender lo mismo — a saber, el estudio de los procesos de significación humana - y que se empeñan en diferenciarse porque se confunde una forma del mismo objeto - la del metaanálisis - con otro objeto enteramente diferente. Debe tenerse presente que el enfoque de ambas disciplinas es el de entender los procesos de significación y que bien podrían hablarse una misma disciplina (semiología) que emplea diferentes metodologías (el análisis meta o el análisis pragmático) para acercarse al objeto de estudio: los signos.

Para mayor claridad, hay que tratar de comprender la hipertrofia conceptual a través de una analogía con un objeto de estudio de las ciencias naturales. Esto es interesante porque, para este tipo de disciplinas, el análisis parte, en muchos casos, de la percepción en tiempo real y, por ende, se hace más simple comprender que un mismo objeto tiene diversas formas de manifestación, antes que considerar que hay diferentes objetos para cada característica o forma de manifestación de uno mismo. Es importante aclarar que esta ilustración analógica no es más que un mecanismo argumentativo que busca facilitar la comprensión del problema abordado y que lo evidenciado en este artículo no pretende, bajo ninguna circunstancia, volver a la vieja discusión de superioridad o inferioridad entre las humanidades y las ciencias naturales, sino evidenciar que las humanidades tienen un poco más de dificultad en el momento de analizar sus objetos de estudio debido precisamente a su profundidad: tratan de entender productos del pensamiento a través del pensamiento mismo. Lo que no las hace en ningún momento inferiores a las naturales, sino distintas. Habiendo hecho estas aclaraciones, se procederá a exponer la analogía.

Como ejemplo, si se trata de estudiar un naranjo, se puede describir como una planta leñosa que se ramifica ampliamente, que tiene espinas y unas hojas ovaladas color verde claro. A estas características habría que añadir el hecho de que la planta florece a partir de unos pequeños brotes blanquecinos y que su fruto es redondo y jugoso. Sin embargo, hay que decir que la posibilidad de análisis solo permitió observar el árbol antes de su etapa fértil. Y, por otro lado, hay que considerar que hubo otros dos analistas: el primero de ellos logró estudiar al naranjo en su etapa de floración y, el segundo, en su momento de cosecha. También debe imaginarse que la nominación hubiese estado relacionada con la forma de las hojas y hubiese tenido como resultado el nombre de «Árbol de las hojas verdo-brillantes redondeadas y puntiagudas». Los otros dos analistas, cada uno por su parte y de acuerdo con su posibilidad de análisis, terminaron por denominar al naranjo: uno como «Árbol de las florecillas blancas» y otro como «Árbol de los frutos naranjas redondeados».

En este simple ejemplo se pueden comprender mucho mejor las consecuencias que surgen del conocimiento derivado de la hipertrofia conceptual de la que se está hablando: un mismo árbol es reconocido como tres árboles diferentes, debido a que los analistas no pudieron contrastar sus estudios para llegar a reconocer que se trataba, en realidad, de una única especie, aquella que responde al nombre de citrus sinensis, comúnmente conocida como naranjo, y que puede presentarse en formas distintas según la etapa de reproducción por la que esté atravesando. Ahora bien, si se quisiera eliminar la hipertrofia en este caso, las tres miradas deberán unirse para definir al naranjo como un árbol de hojas ovalado-puntiagudas de color verde claro, que en una época tiene como característica unas 
La literatura oral: una apología del concepto. Parte I: La falsa analogía

inflorescencias blanquecinas y pequeñas y que, en otro momento, produce un fruto redondeado de color naranja.

De esta manera, se comprendería una única nominalización para el objeto de estudio: el naranjo y las diversas características que acompañan su proceso de existencia. Sin embargo, lo que sucede en los campos esencialmente metaanalíticos, como el de la lingüística, el de la literatura y algunos otros es todo lo contrario: los analistas suelen mantener la nominalización divisoria por características. Como en el caso de la semiótica y la semiología, de las que permanece una concepción diferenciada cuando podría decirse que la semiología abarca el metaanálisis de la producción de sentido (semiótica) a través de la contrastación con los fenómenos empíricos (semiología). Lo anterior equivaldría, en el ejemplo planteado, a sostener que cada naranjo, según su etapa, es un naranjo completamente diferente. Esa tendencia tiende a generar una gran dificultad para entender la dinámica estacional del árbol y, por ende, complejiza la posibilidad de entender el árbol esencialmente.

Esto mismo ocurre con la literatura oral (LO en adelante), cuyo conocimiento ha estado mediado por enormes discrepancias entre teóricos y disciplinas, derivando así en una serie de variados conceptos (hipertrofia conceptual) que tratan de enmarcar, en su respectiva nominalización, lo que representa este objeto de estudio para cada perspectiva. De esta manera, se incurre a menudo en una sobrenominalización que surge del empeño en reconocer diversas entidades en donde solo hay múltiples características de un mismo objeto o en defender con vehemencia diferencias teóricas fácilmente dirimibles a través de una aclaración conceptual que no implique la renominalización de la cosa. Según la hipótesis de esta investigación, todo esto ha llevado a que no se haya podido abordar — con pertinencia, en el entorno teórico del mundo académico contemporáneo — lo que representa el arte literario que se transmite de forma oral. Porque esta hipertrofia conceptual ha distanciado el análisis de la LO de los estudios literarios, la disciplina que esta investigación considera la más pertinente para analizarla.

Por lo tanto, a esta investigación le interesa, sobre todo, demostrar que las otras nominaciones que buscan reemplazar el concepto LO: primero, no están lo suficientemente sustentadas, ya que parten de una falsa analogía; segundo, que hacen que el concepto coincida con valores que no le son los más representativos — lo que varía dependiendo de si el investigador pertenece al campo etnográfico, al historiográfico o al literario - y, tercero, que alejan el objeto de estudio de su característica más representativa: sus rasgos literarios. Así, con la LO ocurre de alguna manera lo mismo que le pasó a la lingüística antes de que Saussure consolidara las bases conceptuales de ese campo de análisis:

[...] no es fácil tener una visión global del fenómeno [...] [y, por otro lado, las] manifestaciones exteriores de la lingüística la han entremezclado con otros campos ajenos [...] [como el etnográfico, el historiográfico, el filológico, etc.]; estos campos encontraron un terreno propicio en la lingǘstica, pero no [contribuyeron a] que esta progresara significativamente (Mejía, Jaramillo \& Pérez, 2019, pp. 93-97).

De la misma manera, en el campo de la LO se han desarrollado investigaciones teóricas que distancian al fenómeno del campo literario y que, aunque llegan a conclusiones importantes para su respectiva área de análisis, no hacen que el conocimiento teórico sobre la misma crezca significativamente. Se considera entonces que la LO merece ser analizada más allá de su mera relación con otros fenómenos sociales, ya que en ella se ven anudadas muchas de las características que configuran al ser humano y a las sociedades, pero que están directa 
La literatura oral: una apología del concepto. Parte I: La falsa analogía

y esencialmente relacionadas con el campo literario. Y, para esto, es importante establecer una propedéutica un poco más definida que, buscando la comprensión teórica de la LO en su esencia particular, sirva de base sólida para la construcción de futuras investigaciones prácticas o específicas, cuyos análisis, alimentados por dicho sustento, se vuelvan más profundos y más claros. Según Greimas y Courtés, «[explicitar] los conceptos por definiciones sucesivas resulta ser [...] la primera tarea de toda construcción metalingüística del teórico» (Greimas \& Courtés, 2006, p. 75).

En este sentido, se puede afirmar que el efecto de la nominalización enmarca las posibilidades de análisis de un objeto de estudio y que, por tanto, no es posible adentrarse en el conocimiento de ninguna cosa del mundo sin discernir — con cierto nivel de claridad — una forma de nominalización que dé los lineamientos pertinentes para abordar la investigación. Ya que se considera que solo a través de una nominalización clara y diferenciada se puede alcanzar un nivel pertinente de comprensión de los fenómenos que se investigan y, porque, como se ha evidenciado, es importante emprender una batalla contra la mencionada hipertrofia conceptual, la cual hace que la posibilidad de comprensión se diluya entre la turbulencia de propuestas inconexas, de propuestas que se empeñan en diferenciar tajantemente lo que no es más que un modus operandi de la misma entidad.

Por estas razones, esta investigación busca analizar las motivaciones y los efectos de la hipertrofia conceptual que ha padecido la LO, una tarea de grandes proporciones que no puede resumirse a un solo artículo académico. Lo que se pretende, entonces, es dividir el problema en sus tres características más relevantes, para dar a cada una de ellas el nivel de análisis que merece. Como ya se mencionó, hay tres cosas negativas derivadas de la hipertrofía conceptual que ha sufrido la LO: primero, esa proliferación nominativa no está bien justificada; segundo, los demás conceptos alejan a la LO de sus características más representativas; tercero, los diferentes nombres que han rebautizado el fenómeno lo han alejado de los estudios literarios, la disciplina más pertinente para analizarlo. En este sentido, la apología del concepto se desarrollará en tres partes. Este artículo pretende ser la primera. Así, lo que se analizará a continuación será la base fundante de la cual surge la hipertrofia conceptual padecida por la LO, al explicarse por qué no está lo suficientemente sustentada y mostrarse además el alcance que tuvo en muchas de las investigaciones desarrolladas para estudiar el fenómeno.

\section{La falacia del punto de partida}

Muchas de las investigaciones teóricas que abordan la LO y que sobreconceptualizan el fenómeno, se basan, para hacerlo, en un argumento falaz que se utilizó para diferenciar tajantemente oralidad y escritura a finales del siglo xx. Dentro de la nueva dialéctica o, como prefieren llamarla Van Eemeren y Grootendorst: «pragmadialéctica», se entiende que el punto de partida de una discusión debe ser un acuerdo común que se derive de un razonamiento lógico válido (2006, p. 167). Es importante tener esto presente en la medida en que solo una investigación que surja de un punto de partida lógico puede ser pertinente. Y, como se ha mencionado, muchos de los trabajos que teorizan sobre la LO parten de un descarrilamiento (derailment) ${ }^{3}$ de la regla ocho (la regla del

3. «[...] las falacias cometidas en el discurso argumentativo se caracterizan como descarrilamientos de las maniobras estratégicas. [...] como violaciones de las reglas para la discusión crítica [...]» (Van Eemeren, 2006, p. 56). 
La literatura oral: una apología del concepto. Parte I: La falsa analogía

esquema argumentativo) para la discusión crítica de Van Eemeren y Grootendorst (2006, p. 180); es decir, han establecido una falacia como punto de partida para conceptualizar sobre la LO. Esta falacia se encuentra en el texto Oralidad y escritura: tecnologías de la palabra ${ }^{4}$, de Walter Ong (2007). Sin embargo, antes de desarrollar el análisis que viene a continuación, es necesario manifestar que, a pesar de que en esta investigación se plantee un desacuerdo con algunas de las propuestas de Ong, no implica, en absoluto, que se deslegitime su trabajo, ya que se lo reconoce como una muy importante fuente para comprender la oralidad y la escritura, a pesar de que extralimite la diferencia que hay entre ellas.

La falacia surge en la década de los ochenta, cuando Walter Ong pretendió establecer que las investigaciones desarrolladas en torno a lo que él denominó «formas de arte verbal» (2007, p. 19), estaban distorsionadas por una perspectiva letrada; es decir, que, al estar tan imbuidas en el medio escrito, no lograban comprender los fenómenos que se desenvolvían en la oralidad, y que lo único que hacían era tratar, infructuosamente por demás, de homologar oralidad y escritura. Es para explicar esta idea que el autor propuso la analogía entre el caballo y el automóvil. Según Ong,

pensar en la tradición oral o en la herencia de los performances, géneros y estilos orales como «literatura oral» sería pensar en los caballos como automóviles sin ruedas [...] Se desarrolla un discurso sobre caballos que siempre se refiere a ellos como «automóviles sin ruedas». [...] Al final, los caballos son solamente lo que no son (Ong, 2007, p. 12$)^{5}$.

Es en este sentido, en el que Ong manifestó que el de LO es un «monstruoso concepto» (2007 p. 11). Por su parte, en esta investigación se considera que, dadas las características especiales que tiene el fenómeno de la LO, esta afirmación no está adecuadamente justificada, puesto que se construye a partir de un evidente paralogismo:

En el caso de argumentos por analogía, una de las preguntas críticas es si realmente se justifica la comparación o si existen diferencias fundamentales. Si la comparación es defectuosa, el argumento por analogía está incorrectamente usado y constituye una falacia de falsa analogía (van Eemeren \& Grootensdort, 2006, pp. 181-182).

Y, por otro lado, que el argumento que busca sustentarse con esta falsa analogía no presenta razones suficientes para eliminar el concepto de LO. En este sentido, lo primero que se debe considerar es que Ong buscaba evidenciar que existe una diferencia insalvable entre oralidad y escritura a partir de dos estrategias argumentativas principales: la analogía y el argumento etimológico. Lo que se hará, entonces, será desestimar la primera de estas estrategias, analizando la propiedad de su comparación; seguidamente, se verificará la asertividad del argumento que intentaba sustentar la mencionada analogía — el cual manifiesta que la oralidad y la escritura son, esencialmente y diametralmente, diferentes-; tercero, se comprobará que el argumento etimológico no tiene sentido en la actualidad y, por último, se planteará una crítica frente a otras ideas que presentó el autor en

4. $\quad$ Orality and Literacy: Technologizing of the Word.

5. «[...] thinking of oral tradition or a heritage of oral performance, genres and styles as "oral literature" is rather like thinking of horses as automobiles without wheels [...] It proceeds to discourse on horses by always referring to them as "wheelless automobiles". $[\ldots]$ In the end, horses are only what they are not» (Ong, 2007 p. 12). 
La literatura oral: una apología del concepto. Parte I: La falsa analogía


y escritura y que, de acuerdo con esto, no había motivo primario para la proliferación de nombres que surgió de esta diferenciación.

\subsection{La falsa analogía}

Se comenzará entonces con la revisión de la analogía propuesta por Ong. Para hacerlo, se debe considerar primero las diferencias entre el automóvil y el caballo. En principio, el automóvil es, esencialmente, una máquina creada por el hombre, del cual puede decirse que es una evolución de la carreta, que funciona a través de la energía del petróleo, que se moviliza por medio de su sistema rotativo, que es inerte, etc., y, funcionalmente, que sirve al hombre para movilizarse. Por su parte, el caballo es, esencialmente, una entidad animal, una creación de la naturaleza, que funciona a través de la tracción animal, que se moviliza por medio del avance de las patas del animal, que está vivo y tiene una voluntad, etc., y que, funcionalmente, le ha servido al hombre para movilizarse.

Ahora, si se ponderan las similitudes y diferencias entre la escritura y la oralidad, puede decirse que, en primer lugar, la escritura es, esencialmente, un sistema creado por el hombre, que es una forma de producción de la lengua humana, que, formalmente, es gráfica y — según el mismo Ong (2007) — subordinativa, analítica, distante, precisa, etc., además de que, funcionalmente, sirve para que el hombre transmita sus ideas. Por su parte, la oralidad es, esencialmente, un sistema creado por el hombre, una forma de producción de la lengua humana, que, formalmente, es fónica y — también de acuerdo con Ong (2007) — es aditiva, redundante, tradicionalista, homeostática, etc., y que, funcionalmente, le sirve al hombre para dar forma a sus ideas.

Como puede verse, cuando Ong utilizó al caballo y al automóvil como ejemplos para establecer las semejanzas y las diferencias entre la escritura y la oralidad, no considera que hay muchas más semejanzas que diferencias entre las dos últimas que entre los dos primeros. Caballo y automóvil se asemejan en que han sido utilizados por el hombre para movilizarse (criterio funcionalista) y se diferencian en que uno es un animal y el otro es una máquina creada por el hombre (criterio esencialista), y se diferencian en la forma en la que se movilizan, en la fuente energética de la que se sirven, en la velocidad que pueden alcanzar y en las distancias que pueden recorrer, etc. En definitiva, su única similitud es que han sido utilizadas (función) por el hombre para movilizarse, pero son esencial y formalmente diferentes.

Por su parte, la escritura y la oralidad se asemejan, esencialmente, en que ambas son una forma de producción

6. $\quad$ Es importante aclarar que lo expresado en este artículo no implica que la oralidad y la escritura sean iguales. La perspectiva desde la que se analiza en esta investigación propone que ambas son muy similares en su esencia, pero formalmente diferentes. Con lo que se puede afirmar que la literatura tiene lugar en ambos sistemas lingüísitcos. Abordar con más profundidad este planteamiento es una tarea necesaria, pero que no corresponde a este trabajo. Sin embargo, una breve aclaración de la similitud a la que se hace referencia fue planteada por Constantin y Saussure (2005) cuando propuso que la lengua — entendida como la oralidad — «Es un conjunto de signos que han sido fijados gracias al convenio de los miembros de la sociedad; los signos evocan las ideas [...]. Asimismo, la escritura es un vasto sistema de signos. Habrá una psicología de los sistemas de signos, esta psicología será una parte de la psicología social, es decir, que no será sino social. Será la misma psicología aplicable a la lengua» (como se citó en Mejía Quijano, Jaramillo Giraldo \& Betancur, 2020, p. 94). Con esta cita se da cuenta de la esencia similar entre la oralidad y la escritura, pues ambas corresponden a ese sistema de signos que remite a la psicología social. Se puede comprender mejor esta idea por medio del trabajo de Mejía Quijano et al. (2020). 
La literatura oral: una apología del concepto. Parte I: La falsa analogía

de la lengua humana y en que ambas son sistemas que han sido creados por el hombre; funcionalmente, en que ambas transmiten ideas a través de palabras o sintagmas y en que ambas hacen referencia a las actividades del hombre; y se diferencian, formalmente, en que una es más estática que la otra, en que una es lineal y la otra es ubicua, en que una es sonora y la otra es visual, etc. Por otro lado, se debe tener presente que el padre de la lingüística afirmó que la una nace de la otra, es decir, que la escritura nace de la oralidad. En este sentido, escritura y oralidad son esencialmente similares, funcionalmente similares, formalmente diferentes, y están tan íntimamente relacionadas que una es la que engendra a la otra, como también el mismo Ong lo manifestó (2007, p. $11)^{7}$. De esta manera, mientras que no hay forma alguna en la que el automóvil haya nacido del caballo o sea una forma diferente de representar el caballo, la escritura sí nació necesariamente de la oralidad y, no solo puede, sino que busca representarla - al menos esa es una de sus funciones- (Mejía et al., 2019). Finalmente, de acuerdo con la distinción de Kant, toda analogía se construye como igualdad de dos relaciones cualitativas (s.f., pos. 2847); de modo que Ong, al saltarse el orden intrínseco de las cosas comparadas, comete una falta contra la lógica. Su analogía carace de los criterios necesarios de correspondencia al equiparar dos órdenes distintos — según un único criterio funcionalista - con dos ordenes semejantes que solo se diferencian en sus aspectos formales.

\subsection{Un argumento sin razones suficientes}

En esta sección, se abordará en profundidad el argumento que propone esta analogía: la diferencia tajante entre la oralidad y la escritura. Quizá si el filólogo estadounidense hubiese propuesto que hablar de literatura oral, desde la perspectiva de la literatura escrita, era como hablar de carretas desde la idea de los automóviles, su análisis hubiera sido más sensato, pues en este caso sí se estarían comparando dos cosas de la misma naturaleza: máquinas construidas por el hombre para movilizarse. Sin embargo, de haberlo hecho de esta manera, su argumento — que distancia diametralmente la oralidad de la escritura - no hubiera tenido sentido, porque es muy sencillo distanciar un caballo y un automóvil en tanto no se asemejan en nada más que en la funcionalidad que han tenido para el hombre; pero no lo es tanto si se habla de un automóvil y una carreta, que esencialmente son semejantes. Por tanto, de haber desarrollado una analogía lógica, para Ong no hubiese sido nada fácil distanciar — tan tajantemente como lo hizo - la oralidad de la escritura, y ahí está la importancia de evidenciar esta falsa analogía: sin ella no se pueden separar sino formalmente la oralidad y la escritura.

Este distanciamiento de Ong también hubiera podido ser un poco más lógico si tanto la oralidad como la escritura estuvieran constituidas esencialmente por materias diferentes, es decir, si fueran esencialmente diferentes - suponiéndose que una fuese un código lingüístico y la otra otro pictórico- . No obstante, en esta investigación se considera que, así como dijo Aristóteles, que la retórica es vástago ${ }^{8}$ de la dialéctica (Trad. en 1962, pos. 13

7. $\quad$ Según Ong (2007), la oralidad secundaria, la que se experimenta en el mundo contemporáneo, nace de la escritura y se nutre constantemente de ella; mientras que la escritura, a su vez, se nutre constantemente de la oralidad secundaria. Un ejemplo de esto puede ser la forma oral que han adquirido los mensajes de texto.

8. «de manera que acontece a la retórica ser como un esqueje de la dialéctica» (Trad. en 1962, pos. 13 402). 
La literatura oral: una apología del concepto. Parte i: La falsa analogía

402), de la misma manera se puede decir que la escritura es vástago de la oralidad y que está hecha de su misma materia: la lengua; a pesar de que se diferencien en su forma están unidas desde su esencia.

Como dice Saussure: «[tenemos] dos sistemas que se corresponden, el de los signos escritos y el de los sonidos» (Mejía et al., 2019, p. 109), con lo que se sigue que «[no] existen dos tipos de palabras (...) la palabra escrita no está coordinada con la palabra hablada, sino subordinada» (Mejía et al., 2019, p. 104). Entonces, debe quedar claro que lo importante no es solo que se haya cometido una falsa analogía, sino que, una vez se elimina ese mecanismo argumentativo, la idea de que la oralidad y la escritura son esencialmente diferentes pierde valor.

Ahora queda demostrar por qué otras de las estrategias argumentativas de Ong siguen siendo poco pertinentes para distanciar tangencialmente la escritura de la oralidad. Por ejemplo, en favor de su argumento y para desestimar a Ruth Finnegan — quien defendía el concepto de literatura oral manifestando que «la "literatura" aunque ideada primeramente por trabajos escritos, simplemente se ha extendido hasta incluir otros fenómenos relacionados $)^{9}$ (Ong, 2007, p. 12)—, Ong utilizó el arma de la etimología: «los conceptos tienen una manera de cargar sus etimologías con ellos para siempre ${ }^{10}$ (p. 12). Sin embargo, ya se ha demostrado reiteradamente que las etimologías pierden su sentido en el momento en el que la fuente primaria se desmotiva ${ }^{11}$ (Guiraud, 1997), que es precisamente lo que le ha pasado al concepto de la literatura.

Por último, para cerrar su propuesta de contraponer diametralmente estas dos formas de producción de la lengua, Ong (2007) cayó en una curiosa contradicción, al manifestar que «la oralidad necesita y está destinada a producir la escritura. [y que] La literacidad [...] es absolutamente necesaria para el desarrollo no solo de la ciencia sino también de la historia, la filosofía $[\ldots]\rangle^{12}$ (p. 14). Con esto, el autor no solo demostró que tanto la oralidad como la escritura están irremediablemente unidas — que nacen una de la otra—, sino también que su análisis parte de un sesgo que definitivamente disminuye la importancia de lo oral frente a lo escrito. Otras de sus afirmaciones que evidencian esto son: «La oralidad no es un ideal y nunca lo fue (...) La literacidad abre posibilidades al mundo de la existencia humana que son inimaginables sin la escritura (...) Nunca he encontrado o escuchado de una cultura oral que no quiera alcanzar la literacidad lo más pronto posible» ${ }^{13}$ (p. 171), entre otras.

Y todo esto lo manifiesta después de que, ya para su tiempo, y gracias a autores que él mismo citó, se había demostrado que no es del todo cierto que los grandes avances de la humanidad nacen de lo escrito. Ya Milman Parry (1971) había demostrado que la literatura nace en la oralidad y — hoy en día — otros, como Santa (2006), han demostrado que la historia se conserva también en la oralidad.

9. " "literature", though devised primarily for works in writing, has simply been extended to included related phenomena.»

10. «concepts have a way of carrying their etimologies with them forever.»

11. «El oscurecimiento de la motivación etimológica. [...] Esta semantización de los valores estilíticos es la fuente más importante de la evolución semántica; alcanza la mayoría de las palabras debilitando sus sentidos» (Guiraud, 1997, p. 76).

12. «Orality needs to produce and is destinated to produce writing. Literacy, as will be seen, is absolutely necessary for the development not only of science, but also of history, philosophy [...]»

13. «Orality is not an ideal and never was [...] Literacy opens possibilities to the world and to human existence unimaginable without writing [...] I have never encountered or heard of an oral culture that does not want to achieve literacy as soon as possible.» 
La literatura oral: una apología del concepto. Parte I: La falsa analogía

Por todo lo anterior, se puede afirmar que la incurrencia en la falsa analogía no es solamente un error lógico, sino la evidencia de que, desde la perspectiva de Ong, no se comprenden claramente las diferencias y las similitudes entre la oralidad y la escritura, en principio porque hay una distancia entre ambas que no está suficientemente sustentada; luego, porque esta idea se sustenta en criterios etimológicos que — para la semántica contemporáneason muy cuestionables y, por último, porque la lectura del autor surgió de un sesgo frente a la importancia y el lugar que tiene la oralidad para la humanidad. Por tanto, es un error de base partir de esta idea para renominalizar un concepto del cual no se ha demostrado de forma eficiente que es, como lo dijo Ong, monstruoso.

\subsection{El alcance de la falacia y la hipertrofia conceptual}

Lo anterior no es tan sorprendente como la difusión que ha tenido este modo de pensar entre diferentes autores que, partiendo de dicha falsa analogía, optan por rebautizar el fenómeno. Esto se evidencia muy frecuentemente en Latinoamérica. Por ejemplo, Diana Toro (2014) dijo que «de acuerdo con Walter Ong, no es pertinente denominar así a la tradición oral, puesto que la raíz littera del término "literatura" indica la presencia de la escritura» (p. 241) y, siguiendo esta idea, la autora adoptó otros términos: «[la] oralitura indígena, la oralitura afrocolombiana y la tradición oral de ascendencia hispánica» (p. 241); por su parte, Ostria González (2001) manifiesta que el sintagma «literatura oral» constituye en sí mismo un oxímoron, ya que, según él, oralidad y escritura son esencialmente contradictorios, tal y como lo propuso Ong; Alba Moya (2009) propuso «la denominación arte oral como alternativa a la literatura oral», porque «[de] acuerdo con Ong, hablar de literatura oral, para referirse a la oralidad, es una monstruosidad» (p. 25); Mato (1990), después de manifestar que no se ocupará del problema conceptual, afirmó que «sería más pertinente hablar del valor literario de ciertas expresiones verbales» (p. 154) refiriéndose a una posible necesidad de cambiar el concepto y, más adelante, cita a Ong como referencia para dar entender por qué esto sería necesario; Domínguez Cáceres (2011), en su reseña sobre Oralidad y escritura, consideró que «Ong promueve una relectura de las tradicionales concepciones erróneas acerca de lo oral como un subsistema de lo escrito o viceversa» (p. 15). Esperando que sea evidencia suficiente, se deja hasta aquí a los autores latinoamericanos, pero es importante manifestar que son muchos más los casos de influencia.

Otras tradiciones también se han permeado del influjo de las propuestas de Ong. Por ejemplo, Miles Foley (1986b) manifestó que admiraba «las nociones de Walter J. Ong en cuanto oralidad y textualidad, [las cuales encuentra] inquietantemente brillantes y llenas de posibilidades ${ }^{14} \gg$ (p. 16) y, en otro de sus textos (1986a), en su reseña al trabajo de Welber Kelber, concluyó que:

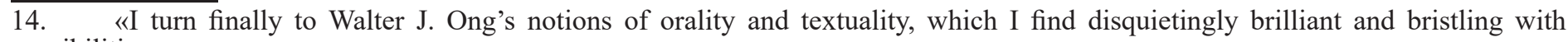
possibilities.» 
La literatura oral: una apología del concepto. Parte I: La falsa analogía

para Kelber lo oral y lo escrito son significativamente diferentes y no hay transición suave entre la una y la otra (...) desde que «el purismo literario» no puede «penetrar el alma de la vida oral» (...) le da un lugar prominente a un modelo más general de la cultura oral sugerido especialmente en los trabajos de Havelock y Ong ${ }^{15}$ (p. 56).

Por su parte, Havelock (1996) declaró que «los lectores de este libro se percatarán de lo que debo a la obra de Walter J. Ong, cuyo magistral estudio Orality and Literacy (1982) me proporcionó la base para la síntesis que aquí se intenta» (p. 17).

Hay otros autores que, aunque no citan directamente el trabajo de Ong, pudieron y parecen estar muy relacionados con lo que este autor estadounidense proponía. Por ejemplo, en uno de sus primeros textos, Lord Albert (2000) expresó que

el término «literatura» presupone el uso de la letra, asume que los trabajos verbales de la imaginación son transmitidos por medio de la escritura y la lectura. La expresión «literatura oral» es obviamente una contradicción entre términos ${ }^{16}$ (p. XXXI).

Y Vladimir Propp (1984) — aunque desde una tradición distante — sostuvo que «el folclor es una forma especial de arte verbal» (p. 14) y que, aunque es similar a la literatura, debe diferenciarse de ella por razones que no son muy diferentes a las de Ong: la diferencia esencial entre la oralidad y la escritura. Las anteriores percepciones se destacan, pues, por su abordaje crítico a la obra de Ong.

Ante estas visiones, se ha dado una proliferación terminológica para tratar de redefinir el fenómeno de literatura oral, cuyos resultados han derivado en términos como folclor, tradición oral, oralitura, etnoliteratura, formas de arte verbal, literatura de tradición oral, entre otros. Sin embargo, también ha habido varias respuestas que optan por criticar la postura de Ong, como la de Freja de la Hoz (2012) que en su trabajo la cuestiona con estas afirmaciones:

«¿Dijo "literatura oral”?». Sí señor, literatura oral. Walter Ong considera fervientemente que «este término [es] sencillamente absurdo... [y que] revela nuestra incapacidad para representar ante nuestro propio espíritu una herencia de material organizado en forma verbal salvo como cierta variante de la escritura, aunque no tenga nada en absoluto que ver con esta última». Es importante aclarar aquí que Walter Ong se refiere a lo verbal sólo en términos de oral y no en términos bajtinianos, en donde lo verbal es toda expresión de la palabra, sea escrita u oral (p. 5).

Por su parte, Colombres (1999, p. 16) mencionó que «Walter Ong propone la expresión verbal arts forms (o sea, artes verbales), como un término unificador de la verdadera literatura (la escrita)» y a partir de esta cita fundamentó su defensa de la literatura oral como un fenómeno esencialmente literario, al afirmar que cambiar el término de lo literario (literatura escrita) por ese capricho de no aceptar el término literatura oral es inviable; además, se evidencia lo esencialmente literario de la LO, en el hecho de que Ong terminó optando por cambiar incluso el término por el de literatura escrita.

15. «For Kelber oral and written are significantly distinct and there is no smooth transition from one to the other. (...) since "literary purism" cannot "penetrate to the soul of oral life". [...] he gives a prominent place to a more general, universal model of oral culture of the type suggested especially in the work of Havelock and Ong.»

16. «The term "literature", presupposing the use of letter, assumes that verbal works of imagination are transmitted by means of writing and reading. The expression "oral literature" is obviously a contradiction in terms.» 
La literatura oral: una apología del concepto. Parte I: La falsa analogía

Por último, se presentarán a aquellos autores que terminaron reconciliándose con la idea de lo literario. Por ejemplo, Lord Albert (1991) se refiirió al fenómeno como

lo que - en todo derecho - llamamos literatura, y hago énfasis en las cualidades positivas y creativas de la literatura oral, la literatura más antigua del mundo humano, y en su significado para "la literatura escrita". Como mi título indica, voy a discutir sobre las palabras antes que sobre la oralidad, las palabras en su forma oral, como eran, y las palabras en su forma escrita ${ }^{17}$ (p. 16).

Y Miles Foley (2012, p. 40) propuso lo siguiente: «El Proyecto de los Caminos usa el término "ágora" para denotar un mercado verbal, un sitio virtual de intercambio, un espacio y nexo público donde las ideas y el conocimiento se comparten a través de cualquier medio que la comunidad haya adoptado como la tecnología por defecto $^{18} \gg$, en referencia a la oralidad y la escritura como dos ágoras, como dos plataformas de la palabra que terminan siendo esencialmente similares.

Lastimosamente, la defensa del término que hacen estos autores, o no es muy directa en algunos, o no ha tenido mucho alcance en otros. Aún hoy, muchas personas siguen partiendo de esa primera parte de Oralidad y escritura para comenzar su proceso de comprensión del fenómeno de la LO, sin preocuparse por la asertividad de la argumentación que allí se desarrolla y sin abordar todo el texto de Ong, para darse cuenta de que esa primera parte no es todo lo que este investigador tenía para decir, ni es lo más relevante de su obra. Y es que es importante insistir en que en este artículo no se criticó la influencia que haya tenido Ong sobre varios autores, pues sin duda la obra del autor sigue siendo una fuente primaria en los análisis que competen a la literatura. Solo puede decirse que la tajante diferencia entre la oralidad y la escritura no está adecuadamente justificada y que es una lástima que tantos autores se hayan dejado llevar por ella y hayan caído en la hipertrofia conceptual debido a esto.

\section{Conclusión}

En este artículo se buscó, pues, evidenciar la naturaleza y las posibles consecuencias negativas de la problemática de la que están sufriendo el fenómeno de la literatura oral y muchas otras disciplinas de las ciencias humanas: la hipertrofia conceptual. Se dio cuenta de la razón principal por la que la LO está siendo sobrenominalizada: la idea de la irremediable distancia entre oralidad y escritura, que tiene como base teórica fundamental el texto de Walter J. Ong (2007) Oralidad y escritura: tecnologías de la palabra. Se demostró, además, que esa diferencia no está adecuadamente fundamentada, debido a que parte de una falsa analogía y a que sin esa estrategia retórica no hay razones suficientes para sustentar aquella diferenciación;

Por otra parte, se dio cuenta de que otras estrategias - como la etimológica - tampoco validan la idea y, finalmente, que el mismo Ong se contradijo al declarar que la oralidad y la escritura son contradictorias, pero

17. «[..] what we rightly call literature, and to stress the positive and creative qualities of oral literature, the oldest literature in the human world, and its significance for "written literature". As my title indicates, I intend to discuss words rather than orality, words in their oral form, as it were, and words in their written form.»

18. «[...] the Pathways Project uses the term "agora" to denote a verbal market place, a virtual site for exchange, a public space and nexus where ideas and knowledge are shared via whatever medium the community has adopted as the default technology.» 
La literatura oral: una apología del concepto. Parte I: La falsa analogía

que aún así nacen la una de la otra; como si del fuego pudiera surgir el hielo. Se evidenció, además, algunas de las obras que se dejaron influenciar por la propuesta diferenciativa de Ong y que terminaron buscando un nombre alternativo para el fenómeno, lo que devino en la hipertrofía conceptual de la que hoy sufre el campo de la literatura oral. Finalmente, se expusieron someramente algunas otras propuestas que defienden el valor esencialmente literario de la LO. Dicho esto, se cumplió la primera parte de la tarea de dedicar una apología al concepto de la literatura oral.

Quedan, sin embargo, otras dos tareas por realizar. En la Parte II, se demostrará que los conceptos que han buscado reemplazar el de literatura oral no son los más pertinentes para nominalizar el fenómeno, porque solo alcanzan a reconocer en él ciertos criterios que no son los más relevantes para su comprensión y que sirven más a la comprensión de la influencia de este fenómeno en diferentes campos sociales. Algunas de esas propuestas son: la de Jan Vansina (1985), que entendió el fenómeno como una condensación del hecho histórico; la de Vladimir Propp (1984) que lo comprende como un asunto esencialmente folclórico; la de Havelock (1996) que se refirió a él como a una «enciclopedia cultural»; la de Daniel Mato (1990), que la analizó desde una perspectiva etnográficoliteraria, entre muchas otras. Cuando se abordan estas investigaciones, se evidenció que sus miradas representan importantes divergencias y que se han desenvuelto con una gran distancia marcada entre ellas, es decir, rara vez se citan unas a otras y esto ha causado que el análisis del fenómeno se desenvuelva de forma sesgada.

Por último, en futuros trabajos se intentará también exponer, en la Parte III, las razones por las cuales los estudios literarios es la disciplina más apropiada para analizar la literatura oral, para lo cual será necesario mostrar la relación intrínseca entre el fenómeno y las producciones literarias, de la cual ya se ha hecho mención someramente en este artículo, sobre todo cuando se presentaron los autores que tarde o temprano terminaron por defender el valor intrínsecamente literario de la LO. Algunos de ellos son: Gonzálo Espino (1999), Ruth Finnegan (2012), Miles Foley (1985, 1986a, 1986b, 1987, 1988, 2011, 2012), Lord Albert (1975, 1991, 2000), Milman Parry (1971), entre otros.

Finalmente, es pertinente reiterar la importancia de realizar esta apología del concepto de la literatura oral, porque, tal y como lo dijo Constantin y Saussure (2005):

[...] una ciencia ha de definirse primero por lo que no es (...) Podemos entonces empezar, no buscando un principio interior (...), sino partiendo desde lo exterior, desde lo negativo, para luego llegar al principio positivo, como el niño que toma consciencia de sí mismo tanteando el entorno (Mejía et al., 2019, p. 93).

Así, esta investigación, la cual tiene un interés teórico frente al fenómeno de la literatura oral, se tiene la intención de comenzar por decir razonadamente lo que no hace parte del fenómeno literario, para concluir lo que, definitivamente, puede considerarse dentro del mismo. 
La literatura oral: una apología del concepto. Parte I: La falsa analogía

\section{Referencias bibliográficas}

1. Aristóteles (1982). Tratados de lógica. Organón I (Trad. de Miguel Candel Sanmartín). Madrid: Editorial Gredos.

2. Colombres, A. (1999). Oralidad y literatura oral. Revista PROA, 17, 83-89.

3. Domínguez Cáceres, R. (2011). Reseña de Walter J. Ong: Oralidad y escritura: tecnologías de la palabra. Libros Básicos en la Historia del Campo Iberoamericano de Estudios en Comunicación, 75, febrero-abril.

4. Espino, G. (1999). La literatura oral o la literatura de tradición oral. Digital: Abya-Yala.

5. Finnegan, R. (2012). Oral Literature in Africa. Cambridge, UK: Open Book Publishers.

6. Freja de la Hoz, A. F. (2012). Romances, coplas y décimas en el Pacífico y el Caribe colombiano: poética oral en Colombia (Tesis de maestría). Bogotá: Universidad Nacional de Colombia.

7. Greimas, A. J. \& Courtés, J. (2006). Semiótica. Diccionario razonado de la teoría del lenguaje. [Sémiotique. Dictionnaire raisonné de la théorie du langage]. (2a reimpresión). Madrid: Gredos.

8. Guiraud, P., \& Hasler, J. A. (1997). La semántica (2a edición). Colombia: Breviarios del Fondo de Cultura Económica.

9. Havelock, A. (1996). The Muse Learns to Write. Reflections on Orality and Literacy from Antiquity to the Present [La musa aprende a Escribir. Reflexiones sobre Oralidad y Escritura de la Antigüedad hasta el Presente]. Barcelona: Paidós.

10. Jason, H. (1968). A Multidimensional Approach to Oral Literature: A Proposal. Current Anthropology. Chicago, USA.

11. Kant, I. (s.f.). Crítica de la razón pura. Kindle Edition.

12. Lord, A. (1975). Perspectives on Recent Work on Oral Literature. In J. J. Duggan (Ed.), Oral Literature: Seven Essays (pp. 1-24). London: Scottish Academic Press.

13. Lord, A. (1991). Epic Singers and Oral Tradition. London: Cornell University.

14. Lord, A. (2000). The Singer of Thales. Cambridge: Harvard University Press.

15. Magrassi, G. \& Rocca, M. (Eds.) (1991). Introducción al folklore. Foster, Chertudi \& Otros. Buenos Aires: Centro Editor de América Latina.

16. Mato, D. (1990). El arte de narrar y la noción de literatura oral. Protopanorama intercultural y problemas epistemológicos (1a edición). Caracas: Universidad Central de Venezuela, Consejo de Desarrollo Científico y Humanístico.

17. Mejía Quijano, C., Jaramillo Giraldo, D. \& Pérez Zapata, A. (Eds.) (2019). El primer curso. Lingüística general de Ferdinand de Saussure, Louis Caille y Albert Riedlinger. Medellín: Editorial Semsa.

18. Mejía Quijano, C., Jaramillo Giraldo, D., \& Betancur, J. L. (2020). Transferencia y contraste. Una metodología semiológica para el aprendizaje del francés por hispanohablantes. Lenguaje, 48(1), 84-123. doi: 10.25100/ lenguaje.v48i1.8575 
La literatura oral: una apología del concepto. Parte I: La falsa analogía

19. Miles Foley, J. (Ed.). (1981). Oral Traditional Literature: A Festschrift for Albert Bates Lord. Columbus: Slavica, rpt. 1983.

20. Miles Foley, J. (1985). Oral-Formulaic Theory and Research: An Introduction and Annotated Bibliography. New York: Garland.

21. Miles Foley, J. (1986a). Oral Tradition. Columbus: Slavica Publishers, Inc.

22. Miles Foley, John (Ed). (1986b). Oral Traditional in Literature. An Interpretation in Context. Columbia: University of Missouri Press.

23. Miles Foley, J. (1987). Man, Muse, and Story: Psychohistorical Patterns in Oral Epic Poetry. Oral Tradition, 2(1). 91-107.

24. Miles Foley, J. (1988). The Theory of Oral Composition and Methodology. Bloomington: Indiana University Press.

25. Miles Foley, J. (2011). Annotated Bibliography of Works. Oral Tradition, 26(2), 677-524.

26. Miles Foley, J. (2012). Oral Tradition and the Internet: Pathways of the Mind. Chicago: University of Illinois Press.

27. Moya, A. (2009). Arte oral del Ecuador. Quito: Ediciones La Tierra.

28. Ong, J. W. (2007). Orality and Literacy. The Technologizing of the Word (1st edition). (7th reprinting). Londres: Routledge.

29. Ostria González, M. (2001). Literatura oral, oralidad ficticia. Estudios Filológicos, 36, 71-80.

30. Parry, M. (1971). The Making of Homeric Verse. In A. Parry (Ed.). Oxford: Clarendon Press.

31. Propp, V. (2009). Morphology of the Folk Text. (20th paperback printing). Austin: University of Texas Press.

32. Propp, V. (1984). Theory \& History of Folklore (Theory and History of Literature). University of Minnesota Press.

33. Santa, E. (2006). La tradición oral como fuente de la investigación histórica. Boletín de Historia y Antigüedades, 93(833), abril-junio, 405-420.

34. Toro Henao, D. (2014). Oralitura y tradición Oral. Una propuesta de análisis de las formas artísticas orales. Lingüística y Literatura, 65, 239-256. Medellín: Universidad de Antioquia.

35. Van Eemeren, F. H. \& Grootendorst, R. (2006). Argumentación, comunicación y falacias. Una perspectiva pragma-dialéctica (2a edición). Santiago: Ediciones Universidad Católica de Chile.

36. Van Eemeren, F. H. (2012). Maniobras estratégicas en el discurso argumentativo. México: Plaza y Valdé Editores.

37. Vansina, J. (1985). Oral Tradition as History. London: University of Wisconsin Press. 